\title{
NEW FAMILIES OF PERIODIC ORBITS FOR A GALACTIC POTENTIAL
}

MARÍA T. DE BUSTOS ${ }^{1}$, JUAN L.G. GUIRAO ${ }^{2}$, JAUME LLIBRE ${ }^{3}$ AND JUAN A. VERA ${ }^{4}$

Abstract. The Hamiltonian system associated to the Hamiltonian

$$
\begin{aligned}
\mathcal{H}= & \frac{1}{2}\left(P_{1}^{2}+P_{2}^{2}+P_{3}^{2}\right)+\frac{1}{2}\left(Q_{1}^{2}+Q_{2}^{2}+Q_{3}^{2}\right) \\
& +\varepsilon\left(Q_{1}^{4}+Q_{2}^{4}+Q_{3}^{4}+a\left(Q_{1}^{2} Q_{2}^{2}+Q_{1}^{2} Q_{3}^{2}+Q_{2}^{2} Q_{3}^{2}\right)\right),
\end{aligned}
$$

where $\varepsilon$ and $a$ are parameters and $\varepsilon$ is small, describes the local motion in the central area of a galaxy. Its dynamics have been study by many authors. Here we find analytically new families of periodic orbits of this Hamiltonian system.

\section{INTRODUCTION AND STATEMENT OF THE MAIN RESULTS}

In this paper we study the families of periodic orbits of a 3-dimensional (or simply 3D) isotropic harmonic oscillator perturbed by a polynomial potential

$$
\mathcal{H}=\frac{1}{2}\left(P_{1}^{2}+P_{2}^{2}+P_{3}^{2}\right)+\frac{1}{2}\left(Q_{1}^{2}+Q_{2}^{2}+Q_{3}^{2}\right)+\varepsilon \mathcal{P}\left(Q_{1}, Q_{2}, Q_{3}\right),
$$

where $\varepsilon$ is a small parameter. The polynomial potential is

$$
\mathcal{P}\left(Q_{1}, Q_{2}, Q_{3}\right)=Q_{1}^{4}+Q_{2}^{4}+Q_{3}^{4}+a\left(Q_{1}^{2} Q_{2}^{2}+Q_{1}^{2} Q_{3}^{2}+Q_{2}^{2} Q_{3}^{2}\right),
$$

where $a \in \mathbb{R}$ is a parameter.

The potential here studied

$$
I I=\frac{1}{2}\left(Q_{1}^{2}+Q_{2}^{2}+Q_{3}^{2}\right)+\varepsilon\left(Q_{1}^{4}+Q_{2}^{4}+Q_{3}^{4}+a\left(Q_{1}^{2} Q_{2}^{2}+Q_{1}^{2} Q_{3}^{2}+Q_{2}^{2} Q_{3}^{2}\right)\right)
$$

is a galactic potential which describes the local motion in the central area of a galaxy. This potential has been studied by many authors, see for instance Deprit and Elipe [4], Caranicolas [3], Elipe and Deprit [5], Elipe [6], Arribas et al. [2], Zotos [10, 11, 12, 13], Zotos and Caranicolas [14], Zotos and Carpintero [15], ...

In the paper of [9] the authors studied analytically the families of periodic orbits of the Hamiltonian (1) with (2) using the averaging theory, and they find several families of periodic orbits. Here we improve the results of [9] finding new families of periodic orbits, also using a result based in the averaging theory. The key point for obtaining these new families of periodic orbits is to work with the Lissajous variables instead of working directly with the cartesian variables $\left(Q_{1}, Q_{2}, Q_{3}, P_{1}, P_{2}, P_{3}\right)$ as in [9].

The 3D Lissajous variables $(L, l, G, g, N, n)$ are defined through the transformation

$$
\mathcal{L}:\left(Q_{1}, Q_{2}, Q_{3}, P_{1}, P_{2}, P_{3}\right) \mapsto(L, G, N, l, g, n): \mathbb{R}^{6} \rightarrow \Omega \times \gamma
$$

2010 Mathematics Subject Classification. Primary 34C10, 34C25.

Key words and phrases. galactic potential, family of periodic orbits, averaging theory. 
given by

$$
\begin{array}{ll}
Q_{1}=\sqrt{G+N} \sin (l+g+n), & P_{1}=\sqrt{G+N} \cos (l+g+n), \\
Q_{2}=\sqrt{L-G} \sin (l-g+n), & P_{2}=\sqrt{L-G} \cos (l-g+n), \\
Q_{3}=\sqrt{L-N} \sin (l+g-n), & P_{3}=\sqrt{L-N} \cos (l+g-n),
\end{array}
$$

where

$$
\Omega=\left\{(L, G, N) \in \mathbb{R}^{3}: L>0,|G|<L,|N|<L\right\}
$$

and $\gamma$ is the torus

$$
\left\{(l, g, n) \in \mathbb{R}^{3} /(l, g, n) \in[0,2 \pi)^{3}\right\} .
$$

The 3D Lissajous transformation is a canonical transformation, i.e. the symplectic structure remains the standard one. In the new coordinates the Hamiltonian (1) becomes

$$
\mathcal{H}=L+\varepsilon \mathcal{P}_{1}(L, l, G, g, N, n) .
$$

where $\mathcal{P}_{1}(L, l, G, g, N, n)$ is the pullback of the 3 D Lissajous transformation with the perturbed polynomial $\mathcal{P}$.

Theorem 1. For $\varepsilon \neq 0$ sufficiently small in the invariant set $\mathcal{H}=h>0$, the Hamiltonian system defined by the Hamiltonian (3) with the perturbation given by (2) has the following new families of $2 \pi$-periodic solutions in the variable $l$.

If $a \in(-6,6) \backslash\{0,2\}$ we have the families

I): $\gamma_{\varepsilon}^{I}(l)=(L(l, \varepsilon), G(l, \varepsilon), g(l, \varepsilon), N(l, \varepsilon), n(l, \varepsilon))$ such that

$$
\lim _{\varepsilon \rightarrow 0} \gamma_{\varepsilon}^{I}(0)=\left(h, \frac{(a+6) h}{18-a}, \frac{k \pi}{2}, \frac{3(2-a) h}{18-a}, \frac{\pi}{4}+\frac{m \pi}{2}\right)
$$

for $k, m=0,1,2,3$. See Figure 1.

II): $\gamma_{\varepsilon}^{I I}(l)=(L(l, \varepsilon), G(l, \varepsilon), g(l, \varepsilon), N(l, \varepsilon), n(l, \varepsilon))$ such that

$$
\lim _{\varepsilon \rightarrow 0} \gamma_{\varepsilon}^{I I}(0)=\left(h, \frac{3(2-a) h}{18-a}, \frac{\pi}{4}+\frac{k \pi}{2}, \frac{(a+6) h}{18-a}, \frac{m \pi}{2}\right)
$$

for $k, m=0,1,2,3$. See Figure 2.

III): $\gamma_{\varepsilon}^{I I I}(l)=(L(l, \varepsilon), G(l, \varepsilon), g(l, \varepsilon), N(l, \varepsilon), n(l, \varepsilon))$ such that

$$
\lim _{\varepsilon \rightarrow 0} \gamma_{\varepsilon}^{I I I}(0)=\left(h, \frac{(a+6) h}{18-a}, \frac{\pi}{4}+\frac{k \pi}{2}, \frac{(a+6) h}{18-a}, \frac{\pi}{4}+\frac{m \pi}{2}\right)
$$

for $k, m=0,1,2,3$. See Figure 3.

If $a \in \mathbb{R} \backslash\{0,2\}$ we have the families

IV): $\gamma_{\varepsilon}^{I}(l)=(L(l, \varepsilon), G(l, \varepsilon), g(l, \varepsilon), N(l, \varepsilon), n(l, \varepsilon))$ such that

$$
\lim _{\varepsilon \rightarrow 0} \gamma_{\varepsilon}^{I}(0)=\left(h, \frac{h}{3}, \frac{k \pi}{2}, \frac{h}{3}, \frac{m \pi}{2}\right)
$$

for $k, m=0,1,2,3$. See Figure 4 .

Theorem 1 is proved in section 2 .

If we write the periodic orbits described in Theorem 1 in Lissajous coordinates $(L, l, G, g, N, n)$ in cartesian coordinates $\left(Q_{1}, Q_{2}, Q_{3}, P_{1}, P_{2}, P_{3}\right)$ we obtain Table 1 .

From Table 1 is easily to obtain the implicit equations of two of the periodic orbits of the family I for $\varepsilon=0$, which are given by the intersection of the elliptic cylinder 


\begin{tabular}{|c|c|c|c|}
\hline \multirow{12}{*}{ Families } & \multirow{3}{*}{ I } & $Q_{1}(t, \varepsilon)$ & $\begin{array}{c}-\Gamma_{1} \sin \left(t-\frac{\pi}{4}\right)+O(\varepsilon) \\
\Gamma_{1} \sin \left(t+\frac{\pi}{4}\right)+O(\varepsilon)\end{array}$ \\
\hline & & $Q_{2}(t, \varepsilon)$ & $\begin{array}{l}\mp \Gamma_{1} \sin \left(t-\frac{\pi}{4}\right)+O(\varepsilon) \\
\mp \Gamma_{1} \sin \left(t+\frac{\pi}{4}\right)+O(\varepsilon)\end{array}$ \\
\hline & & $Q_{3}(t, \varepsilon)$ & $\begin{array}{l}\mp \Gamma_{2} \sin \left(t+\frac{\pi}{4}\right)+O(\varepsilon) \\
\mp \Gamma_{2} \sin \left(t-\frac{\pi}{4}\right)+O(\varepsilon)\end{array}$ \\
\hline & \multirow{3}{*}{ II } & $Q_{1}(t, \varepsilon)$ & $\begin{array}{l}-\Gamma_{1} \sin \left(t-\frac{\pi}{4}\right)+O(\varepsilon) \\
-\Gamma_{1} \sin \left(t+\frac{\pi}{4}\right)+O(\varepsilon)\end{array}$ \\
\hline & & $Q_{2}(t, \varepsilon)$ & $\begin{array}{l}\mp \Gamma_{2} \sin \left(t+\frac{\pi}{4}\right)+O(\varepsilon) \\
\mp \Gamma_{2} \sin \left(t-\frac{\pi}{4}\right)+O(\varepsilon)\end{array}$ \\
\hline & & $Q_{3}(t, \varepsilon)$ & $\begin{array}{l}\mp \Gamma_{1} \sin \left(t-\frac{\pi}{4}\right)+O(\varepsilon) \\
\mp \Gamma_{1} \sin \left(t+\frac{\pi}{4}\right)+O(\varepsilon)\end{array}$ \\
\hline & \multirow{3}{*}{ III } & $Q_{1}(t, \varepsilon)$ & $\begin{array}{l}-\Gamma_{2} \cos t+O(\varepsilon) \\
-\Gamma_{2} \sin t+O(\varepsilon)\end{array}$ \\
\hline & & $Q_{2}(t, \varepsilon)$ & $\begin{array}{l}\mp \Gamma_{1} \sin t+O(\varepsilon) \\
\mp \Gamma_{1} \cos t+O(\varepsilon)\end{array}$ \\
\hline & & $Q_{3}(t, \varepsilon)$ & $\begin{array}{l}\mp \Gamma_{1} \sin t+O(\varepsilon) \\
\mp \Gamma_{1} \cos t+O(\varepsilon)\end{array}$ \\
\hline & \multirow{3}{*}{ IV } & $Q_{1}(t, \varepsilon)$ & $\begin{array}{l}-\Gamma \cos t+O(\varepsilon) \\
-\Gamma \sin t+O(\varepsilon)\end{array}$ \\
\hline & & $Q_{2}(t, \varepsilon)$ & $\begin{array}{l} \pm \Gamma \cos t+O(\varepsilon) \\
\mp \Gamma \sin t+O(\varepsilon)\end{array}$ \\
\hline & & $Q_{3}(t, \varepsilon)$ & $\begin{array}{l} \pm \Gamma \cos t+O(\varepsilon) \\
\mp \Gamma \sin t+O(\varepsilon)\end{array}$ \\
\hline
\end{tabular}

TABLE 1. The orbits of Theorem 1 in cartesian coordinates where $\Gamma=\sqrt{\frac{2 h}{3}}, \Gamma_{1}=\sqrt{\frac{2(a-6) h}{a-18}}$ and $\Gamma_{2}=\sqrt{\frac{2(a+6) h}{18-a}}$.

$\frac{Q_{1}^{2}}{\Gamma_{1}^{2}}+\frac{Q_{3}^{2}}{\Gamma_{2}^{2}}=1$ with the planes $Q_{1}= \pm Q_{2}$. Similarly for the other periodic orbits of the family I, see Figure 1.

Again from Table 1 it follows that the family II comes from the intersection of the elliptic cylinder $\frac{Q_{1}^{2}}{\Gamma_{1}^{2}}+\frac{Q_{2}^{2}}{\Gamma_{2}^{2}}=1$ with the planes $Q_{1}= \pm Q_{3}$. Similarly for the other periodic orbits of the family I, see Figure 2 .

The implicit equations of two periodic orbits of the family III are given by the intersection of the elliptic cylinder $\frac{Q_{1}^{2}}{\Gamma_{2}^{2}}+\frac{Q_{2}^{2}}{\Gamma_{1}^{2}}=1$ with the elliptic cylinder $\frac{Q_{1}^{2}}{\Gamma_{2}^{2}}+\frac{Q_{3}^{2}}{\Gamma_{1}^{2}}=$ 1. Similarly for the other periodic orbits of this family, see Figure 3.

The implicit equation for the orbits of the family IV are $Q_{1}= \pm Q_{2}= \pm Q_{3}$, see Figure 4.

We must mention that in the paper [9] three more additional families of periodic orbits of the Hamiltonian system defined by the Hamiltonian (3) with the perturbation given by (2) were found in our notation they are:

If $a \in \mathbb{R} \backslash\{0,2\}$ we have the families 
V): $\gamma_{\varepsilon}^{I I}(l)=(L(l, \varepsilon), G(l, \varepsilon), g(l, \varepsilon), N(l, \varepsilon), n(l, \varepsilon))$ such that

$$
\lim _{\varepsilon \rightarrow 0} \gamma_{\varepsilon}^{I I}(0)=\left(h, \frac{h}{3}, \frac{\pi}{6}+\frac{k \pi}{2}, \frac{h}{3}, \frac{\pi}{3}+\frac{m \pi}{2}\right)
$$

for $k, m=0,1,2,3$.

VI): $\gamma_{\varepsilon}^{I I I}(l)=(L(l, \varepsilon), G(l, \varepsilon), g(l, \varepsilon), N(l, \varepsilon), n(l, \varepsilon))$ such that

$$
\lim _{\varepsilon \rightarrow 0} \gamma_{\varepsilon}^{I I I}(0)=\left(h, \frac{h}{3}, \frac{\pi}{3}+\frac{k \pi}{2}, \frac{h}{3}, \frac{\pi}{3}+\frac{m \pi}{2}\right)
$$

for $k, m=0,1,2,3$.

If $a \in(-\infty,-6] \cup[3,+\infty)$ we have the families

VII): $\gamma_{\varepsilon}^{V I I, \pm}(l)=\left(L(l, \varepsilon), G(l, \varepsilon), g(l, \varepsilon), N(l, \varepsilon), n^{ \pm}(l, \varepsilon)\right)$ such that

$$
\lim _{\varepsilon \rightarrow 0} \gamma_{\varepsilon}^{V I I, \pm}(0)=\left(h, 0, \frac{k \pi}{2}, h, \pm \frac{1}{2} \arctan \left(\sqrt{\frac{2(a-3)}{a+6}}\right)\right)
$$

for $k=0,1,2,3$.

We remark that the periodic orbits corresponding to the families V, VI and VII found in [9] are rectilinear or circular.

In short, from Theorem 1 and the mentioned results of [9] it follows the next result.

Corollary 2. For $\varepsilon \neq 0$ sufficiently small in the invariant set $\mathcal{H}=h>0$, the Hamiltonian system defined by the Hamiltonian (3) with the perturbation given by (2) has the following periodic orbits

- $I V, V, V I, V I I$ if $a \in(-\infty,-6)$,

- $I V, V, V I$ if $a=-6$,

- $I, I I, I I I, I V, V, V I$ if $a \in(-6,3) \backslash\{0,2\}$,

- $I, I I, I I I, I V, V, V I, V I I$ if $a \in[3,6)$,

- $I V, V, V I, V I I$ if $a \in[6, \infty)$.

The linear stability or instability of the families of periodic solutions described in Corollary 2 are given in the next result.

Theorem 3. The stability or instability of the families of periodic orbits $\gamma_{\varepsilon}^{\mathcal{I}}(l)$ with $\mathcal{I} \in\{I, I I, I I I, I V, V, V I, V I I\}$ is described in what follows.

(1) The families $\gamma_{\varepsilon}^{I}(l), \gamma_{\varepsilon}^{I I}(l)$ and $\gamma_{\varepsilon}^{I I I}(l)$ are unstable if $a \in(-6,6) \backslash\{0,2\}$.

(2) The family $\gamma_{\varepsilon}^{I V}(l)$ is linearly stable if $a \in(-\infty, 0) \cup(2,+\infty)$ and unstable if $a \in(0,2)$.

(3) The families $\gamma_{\varepsilon}^{V}(l)$ and $\gamma_{\varepsilon}^{V I}(l)$ are linearly stable if $a \in(0,2) \cup(2,4)$ and unstable if $a \in(-\infty, 0) \cup(4,+\infty)$.

(4) The family $\gamma_{\varepsilon}^{V I I}(l)$ is unstable.

Theorem 3 is proved in section 3 .

Proposition 4. For $a=0$ and $a=2$ the Hamiltonian system associated to the Hamiltonian (1) with potential (2) is separable in cartesian and symplectic spherical coordinates.

The proof of Proposition 4 is given in section 2 . 

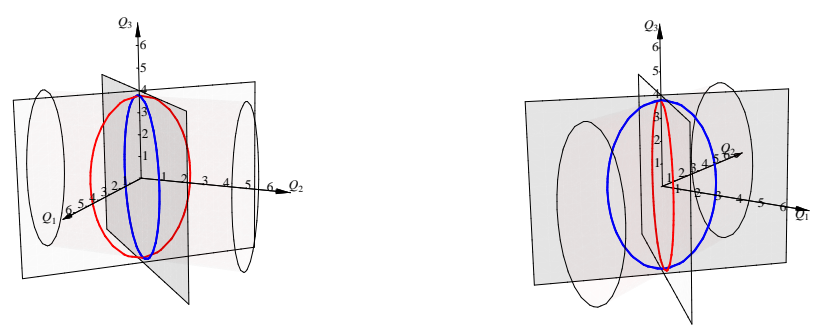

FIGURE 1. Elliptic periodic orbits of the families I.
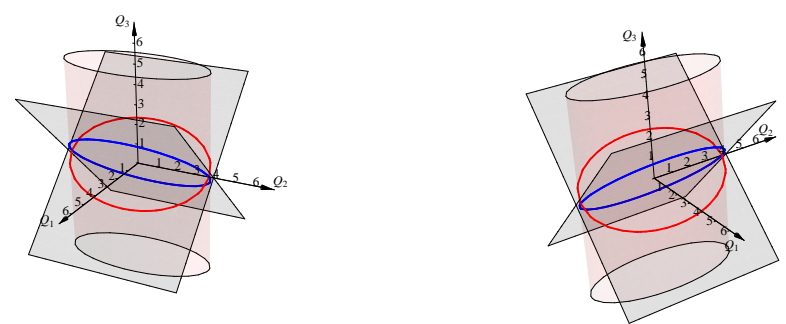

FIGURE 2. Elliptic periodic orbits of the families II.
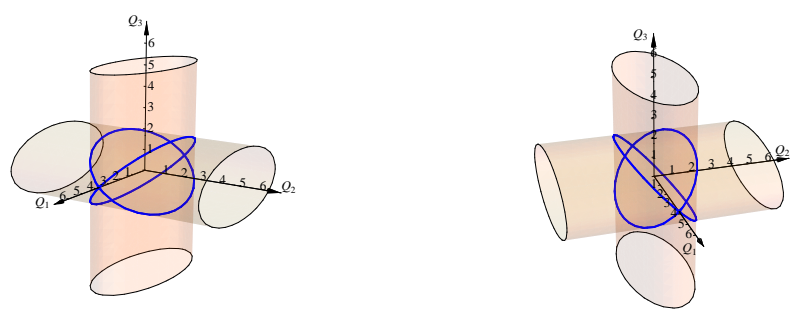

FIGURE 3. Elliptic periodic orbits of the families III.

\section{Proof of Main Result}

First we shall prove Proposition 4.

Proof of Proposition 4. Clearly if $a=0$ the Hamiltonian system associated to the Hamiltonian (1) with potential (2) splits into three separated differential systems in the coordinates $\left(Q_{i}, P_{i}\right)$ for $i=1,2,3$.

For $a=2$ the Hamiltonian $\mathcal{H}$ becomes

$$
\mathcal{H}=\frac{1}{2}\left(P_{1}^{2}+P_{2}^{2}+P_{3}^{2}\right)+\frac{1}{2}\left(Q_{1}^{2}+Q_{2}^{2}+Q_{3}^{2}\right)+\varepsilon\left(Q_{1}^{2}+Q_{2}^{2}+Q_{3}^{2}\right)^{2}
$$



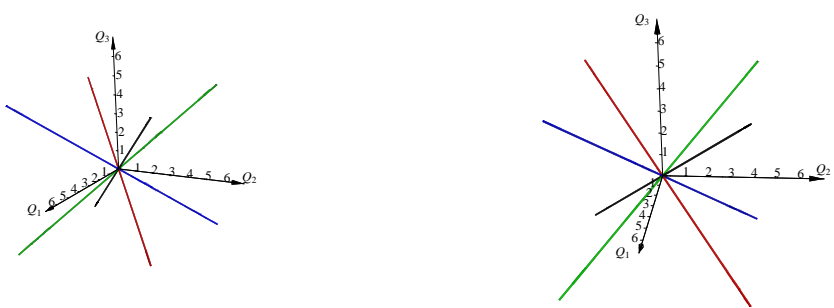

FIGURE 4. Rectilinear periodic orbits of the families IV.

and using symplectic spherical coordinates it writes

$$
\mathcal{H}=\frac{1}{2}\left(p_{r}^{2}+\frac{p_{\theta}^{2}}{r^{2}}+\frac{p_{\phi}^{2}}{r^{2} \sin ^{2} \theta}\right)+\frac{1}{2} r^{2}+\varepsilon r^{4} .
$$

Note that $\mathcal{H}, p_{\theta}$ and $p_{\phi}$ are integrals in involution and consequently the Hamiltonian system associated to the Hamiltonian $\mathcal{H}$ with $a=2$ is completely integrable.

We define the averaged function of $\mathcal{P}$ with respect to the angle $l$, i.e.

$$
\langle\mathcal{P}\rangle=\frac{1}{2 \pi} \int_{0}^{2 \pi} \mathcal{P}(L, l, G, g, N, n) d l
$$

and the functions

$$
\begin{array}{ll}
f_{1}(G, g, N, n)=-\frac{\partial\langle\mathcal{P}\rangle}{\partial g}, & f_{3}(G, g, N, n)=-\frac{\partial\langle\mathcal{P}\rangle}{\partial n} \\
f_{2}(G, g, N, n)=\frac{\partial\langle\mathcal{P}\rangle}{\partial G}, & f_{4}(G, g, N, n)=\frac{\partial\langle\mathcal{P}\rangle}{\partial N} .
\end{array}
$$

We denote by $\mathbf{p}_{0}=\left(G_{0}, g_{0}, N_{0}, n_{0}\right)$ a solution of the system

$$
f_{i}(G, g, N, n)=0 \text { for } i=1,2,3,4,
$$

satisfying that

$$
\Delta=\operatorname{det}\left(\left.\frac{\partial\left(f_{1}, f_{2}, f_{3}, f_{4}\right)}{\partial(G, g, N, n)}\right|_{\mathbf{p}=\mathbf{p}_{0}}\right) \neq 0 .
$$

The next result follows directly from Theorem 1 of [7].

Theorem 5. For $\varepsilon \neq 0$ sufficiently small and for each solution $\left(G_{0}, g_{0}, N_{0}, n_{0}\right)$ of system (4) satisfying (5), the Hamiltonian system defined by the Hamiltonian (3) in the invariant set $\mathcal{H}=h>0$, has a $2 \pi$-periodic solution

$$
\gamma_{\varepsilon}(l)=(L(l, \varepsilon), G(l, \varepsilon), g(l, \varepsilon), N(l, \varepsilon), n(l, \varepsilon))
$$

in the variable $l$ such that

$$
\lim _{\varepsilon \rightarrow 0} \gamma_{\varepsilon}(0)=\left(h, G_{0}, g_{0}, N_{0}, n_{0}\right) .
$$

Moreover, the linear stability or instability of the Poincaré map associated to the periodic solution $\gamma_{\varepsilon}(l)$ is given by the stability or instability of the equilibrium point 
$\left(G_{0}, g_{0}, N_{0}, n_{0}\right)$ of the differential system

$$
\begin{aligned}
\frac{d G}{d l} & =-\varepsilon f_{1}(G, g, N, n), \quad \frac{d N}{d l}=-\varepsilon f_{3}(G, g, N, n), \\
\frac{d g}{d l} & =\varepsilon f_{2}(G, g, N, n), \quad \frac{d n}{d l}=\varepsilon f_{4}(G, g, N, n) .
\end{aligned}
$$

The condition that the determinant $\Delta \neq 0$ in Theorem 5 implies that the averaging theory only can detect periodic orbits which are isolated in the set of all periodic orbits of the differential system. This is the reason that we restrict our study of the periodic orbits of the Hamiltonian system associated to the Hamiltonian (1) with (2) to each level $H=h>0$. We recall that generically the periodic orbits of a non completely integrable Hamiltonian system form cylinders parameterized by $h$, this is the reason that we can find periodic orbits in the levels $H=h>0$ of our Hamiltonian system using the averaging theory, when the Hamiltonian system is not completely integrable, because in this situation the periodic orbits in each level $H=h$ are isolated in the set of all periodic orbits of contained in that level. On the other hand, the fact that we cannot find periodic orbits when $a \in\{0,2\}$ using the averaging theory indicates that for these values of the parameter $a$ the Hamiltonian system can be completely integrable, and consequently their periodic solutions are not isolated in the set of all periodic solutions by the Liouville-Arnold Theorem, and this is the case as it is proved in Proposition 4. For more details on the results stated in these paragraph see the books [1] and [8], the first for the results on Hamiltonian systems and the second for the results on averaging theory.

Solving $H=h$ with respect to the variable $L$, where $H$ is given in (3) and (2), we get $L=h+O(\varepsilon)$. Since we shall study the periodic orbits of the Hamiltonian system, associated to the Hamiltonian (3) with (2), in the level $H=h$ we substitute in what follows $L$ by $h+O(\varepsilon)$.

Applying the 3D-Lissajous transformation to the function $\mathcal{P}\left(Q_{1}, Q_{2}, Q_{3}\right)$ given in (2), it becomes $\mathcal{P}=\mathcal{P}(l, G, g, N, n)$ equal to

$$
\begin{aligned}
\mathcal{P}= & -a(G-h)(G+N) \sin ^{2}(g-l-n) \sin ^{2}(g+l+n)+(h-N) \sin ^{2}(g+l-n) \\
& +a(G+N)(h-N) \sin ^{2}(g+l-n) \sin ^{2}(g+l+n)+(G-h)^{2} \sin ^{4}(g-l-n) \\
& +(G+N)^{2} \sin ^{4}(g+l+n)+(h-N)^{2} \sin ^{4}(g+l-n) .
\end{aligned}
$$

Therefore the averaged function $\langle\mathcal{P}\rangle=\langle\mathcal{P}\rangle(G, g, N, n)$, is

$$
\begin{aligned}
\langle\mathcal{P}\rangle= & \frac{1}{8}(a((h-G)(h-N)(\cos (4(g-n))+2)(\cos (4 g)+2)(h-G)(G+N) \\
& +(G+N)(h-N)(\cos (4 n)+2))+3(G-h)^{2}+3(G+N)^{2}+3(h-N)^{2} .
\end{aligned}
$$

The functions $f_{i}=f_{i}(G, g, N, n)$ for $i=1,2,3,4$ of the differential system (6) are

$$
\begin{aligned}
f_{1} & =-\frac{1}{2} a(G-h)[(G+N) \sin (4 g)+(h-N) \sin (4(g-n))], \\
f_{2} & =\frac{1}{8}[(h-2 G-N)(2 a-6+a \cos (4 g))+2 a(h-N) \sin (2 g) \sin (2 g-4 n)], \\
f_{3} & =\frac{1}{2} a(h-N)[(G-h) \sin (4(g-n))+(G+N) \sin (4 n)], \\
f_{4} & =\frac{1}{8}[(h-G-2 N)(2 a-6+a \cos (4 n))+2 a(G-h) \sin (2 n) \sin (4 g-2 n)] .
\end{aligned}
$$

We need the following technical proposition for proving Theorem 1. 
Proposition 6. Assume that $h>0$. Then we have the following solutions $\left(G_{0}, g_{0}, N_{0}, n_{0}\right)$ of the system $f_{i}(G, g, N, n)=0$ for $i=1,2,3,4$ satisfying that $\Delta \neq 0$.

If $a \in(-6,6) \backslash\{0,2\}$ we have the solutions:

\begin{tabular}{|c|c|c|c|c|c|}
\hline & $G_{0}$ & $g_{0}$ & $N_{0}$ & $n_{0}$ & $\Delta$ \\
\hline I & $\frac{(a+6) h}{18-a}$ & $\frac{k \pi}{2}$ & $\frac{3(2-a) h}{18-a}$ & $\frac{\pi}{4}+\frac{m \pi}{2}$ & $\frac{a^{2}(a-2)^{2}\left(a^{2}-36\right)(a+6) h^{4}}{(18-a)^{3}}$ \\
\hline II & $\frac{3(2-a) h}{18-a}$ & $\frac{\pi}{4}+\frac{k \pi}{2}$ & $\frac{(a+6) h}{18-a}$ & $\frac{m \pi}{2}$ & $\frac{a^{2}(a-2)^{2}\left(a^{2}-36\right)(a+6) h^{4}}{(18-a)^{3}}$ \\
\hline III & $\frac{(a+6) h}{18-a}$ & $\frac{\pi}{4}+\frac{k \pi}{2}$ & $\frac{(a+6) h}{18-a}$ & $\frac{\pi}{4}+\frac{m \pi}{2}$ & $\frac{a^{2}(a-2)^{2}\left(a^{2}-36\right)(a+6) h^{4}}{(18-a)^{3}}$ \\
\hline
\end{tabular}

If $a \in \mathbb{R} \backslash\{0,2\}$ we have the solutions

\begin{tabular}{|c|c|c|c|c|c|}
\hline & $G_{0}$ & $N_{0}$ & $g_{0}$ & $n_{0}$ & $\Delta$ \\
\hline IV & $\frac{h}{3}$ & $\frac{h}{3}$ & $\frac{k \pi}{2}$ & $\frac{m \pi}{2}$ & $\frac{a^{2}(a-2)^{2} h^{4}}{4}$ \\
\hline
\end{tabular}

where $k$ and $m$ vary in the set $\{0,1,2,3\}$.

Proof. If we do at the equations (7) the change of variables $\alpha=G+N, \beta=L-N$ and $\gamma=L-G$, we can rewrite these equations as a quasi-linear system of equations for the unknowns $\alpha, \beta, \gamma$, i.e.

$$
\begin{aligned}
f_{1}= & \alpha \sin (4 g)+\beta \sin (4 g-4 n)=0, \\
f_{2}= & \alpha(6-2 a-a \cos (4 g))+2 a \beta \sin (2 g) \sin (2 g-4 n) \\
& +\gamma(-6+2 a+a \cos (4 g))=0, \\
f_{3}= & \beta(\alpha \sin (4 n)-\gamma \sin (4 g-4 n))=0, \\
f_{4}= & \alpha(6-2 a-a \cos (4 n))+\beta(-6+2 a+a \cos (4 n)) \\
& -2 a \gamma \sin (2 n) \sin (4 g-2 n)=0 .
\end{aligned}
$$

The only equation that it is nonlinear is the third. But it vanishes if and only if either $\beta=0$ or $\alpha \sin (4 n)-\gamma \sin (4 g-4 n)=0$. In the first case, we obtain a linear system $f_{i}=0, i=1,2,4$. In the second, dividing $f_{3}$ by $\beta$, we have another linear system, always in the variables $\alpha, \beta, \gamma$. So, the problem of finding the solutions of the system $f_{i}(G, g, N, n)=0$ for $i=1,2,3,4$ has turned on the problem of finding the solutions of two different linear systems. Now we study the solutions of these two systems.

If $\beta=0$, the linear system writes

$$
\left(\begin{array}{cc}
\sin (4 g) & 0 \\
6-2 a-a \cos (4 g) & -6+2 a+a \cos (4 g) \\
6-2 a-a \cos (4 g) & -2 a \sin (2 n) \sin (4 g-2 n)
\end{array}\right)\left(\begin{array}{l}
\alpha \\
\gamma
\end{array}\right)=\left(\begin{array}{l}
0 \\
0 \\
0
\end{array}\right)
$$

This system has nontrivial solutions when all the minors of second order vanish, that is

$$
\begin{aligned}
(\cos (4 g) a+2 a-6)(a \cos (4 n)+2(\sin (4 g-2 n) \sin (2 n) a+a-3)) & =0 \\
\sin (4 g)(\cos (4 g) a+2 a-6) & =0, \\
\sin (2 n) \sin (4 g-2 n) & =0 .
\end{aligned}
$$

Only four of the eight real solutions of this system satisfy $f_{i}(G, g, N, n)=0$ for $i=1,2,3,4$, namely

$$
\left(G_{0}, g_{0}, N_{0}, n_{0}\right)=\left(0, \frac{k \pi}{2}, L, \pm \frac{1}{2} \arctan \left(\sqrt{\frac{2(a-3)}{a+6}}\right)\right), \quad k=0,1,2,3,
$$

when $a \notin[-6,3)$. These solution corresponds to the solutions (VII) already found in $[9]$. 
We assume now that $\beta \neq 0$. If we interchange the functions $f_{2}$ y $f_{3}$, we obtain the homogeneous linear system

where $A$ is the matrix

$$
A\left(\begin{array}{l}
\alpha \\
\beta \\
\gamma
\end{array}\right)=\left(\begin{array}{l}
0 \\
0 \\
0 \\
0
\end{array}\right)
$$

$$
\left(\begin{array}{ccc}
\sin (4 g) & \sin (4 g-4 n) & 0 \\
\sin (4 n) & 0 & -\sin (4 g-4 n) \\
6-2 a-a \cos (4 g) & -a(\cos (4 n)-\cos (4 g-4 n)) & -6+2 a+a \cos (4 g) \\
6-2 a+a \cos (4 n) & -6+2 a+a \cos (4 n) & a(\cos (4 g)-\cos (4 g-4 n))
\end{array}\right) .
$$

This system has nontrivial solution when all the minors of third order vanish. In this case, we obtain the following solutions $\left(G_{0}, g_{0}, N_{0}, n_{0}\right)$ satisfying (7)

\begin{tabular}{|c|c|c|c|c|}
\hline & $G_{0}$ & $N_{0}$ & $g_{0}$ & $n_{0}$ \\
\hline I & $\frac{(a+6) L}{18-a}$ & $\frac{k \pi}{2}$ & $\frac{3(2-a) L}{18-a}$ & $\frac{\pi}{4}+\frac{m \pi}{2}$ \\
\hline II & $\frac{3(2-a) L}{18-a}$ & $\frac{\pi}{4}+\frac{k \pi}{2}$ & $\frac{(a+6) L}{18-a}$ & $\frac{m \pi}{2}$ \\
\hline III & $\frac{(a+6) L}{18-a}$ & $\frac{\pi}{4}+\frac{k \pi}{2}$ & $\frac{(a+6) L}{18-a}$ & $\frac{\pi}{4}+\frac{m \pi}{2}$ \\
\hline
\end{tabular}

For I, II and III the value of the Jacobian $\Delta$ is $\frac{a^{2}(a-2)^{2}\left(a^{2}-36\right) L^{4}}{(18-a)^{3}}$ and is not zero for $a \neq-6$ and 6 .

For $\beta \neq 0$ there are also the solutions (V) and (VI) already studied in [9].

The restrictions on the parameter $a$ in the statement of Proposition 6 are clear when we compute the periodic orbits associated to the solutions of system (7) using Theorem 5, see the caption of Table 1. 5.

Finally the proof of Theorem 1 follows directly from Proposition 6 and Theorem

\section{Proof of Theorem 3}

By Theorem 5 the stability or instability of the periodic solution $\gamma_{\varepsilon}^{\mathcal{I}}(l)$ with $\mathcal{I}$ $\in\{I, I I, I I I, I V, V, V I, V I I\}$ is given by the stability or instability of the corresponding equilibrium point $\mathbf{p}_{0}=\left(G_{0}, g_{0}, N_{0}, n_{0}\right)$ of system (6) which provides the initial condition of the periodic solution.

The matrix $\mathcal{M}_{\gamma_{\varepsilon}^{\mathcal{I}}(l)}=\left(\left.\frac{\partial\left(f_{1}, f_{2}, f_{3}, f_{4}\right)}{\partial(G, g, N, n)}\right|_{\mathbf{p}=\mathbf{p}_{0}}\right)$ is given by

$$
\begin{gathered}
\mathcal{M}_{\gamma_{\varepsilon}^{I}(l)}=\left(\begin{array}{cccc}
0 & \frac{16 a^{2}(a-6) h^{2}}{(a-18)^{2}} & 0 & -\frac{8 a\left(a^{2}-36\right) h^{2}}{(a-18)^{2}} \\
-\frac{3(a-2)}{4} & 0 & -\frac{3(a-2)}{8} & 0 \\
0 & -\frac{8 a\left(a^{2}-36\right)^{2}}{(a-18)^{2}} & 0 & \frac{16 a\left(a^{2}-36\right) h^{2}}{(a-18)^{2}} \\
-\frac{3(a-2)}{8} & 0 & \frac{6-a}{4} & 0
\end{array}\right) \\
\mathcal{M}_{\gamma_{\varepsilon}^{I I}(l)}=\left(\begin{array}{cccc}
0 & \frac{16 a\left(a^{2}-36\right) h^{2}}{(a-18)^{2}} & 0 & -\frac{8 a\left(a^{2}-36\right) h^{2}}{(a-18)^{2}} \\
\frac{6-a}{4} & 0 & -\frac{3(a-2)}{8} & 0 \\
0 & -\frac{8 a\left(a^{2}-36\right) h^{2}}{(a-18)^{2}} & 0 & \frac{16 a^{2}(a-6) h^{2}}{(a-18)^{2}} \\
-\frac{3(a-2)}{8} & 0 & -\frac{3(a-2)}{4} & 0
\end{array}\right)
\end{gathered}
$$




$$
\begin{aligned}
& \mathcal{M}_{\gamma_{\varepsilon}^{I I I}(l)}=\left(\begin{array}{cccc}
0 & \frac{16 a^{2}(a-6) h^{2}}{(a-18)^{2}} & 0 & -\frac{8 a(a-6)^{2} h^{2}}{(a-18)^{2}} \\
\frac{6-a}{4} & 0 & \frac{a+6}{8} & 0 \\
0 & -\frac{8 a(a-6)^{2} h^{2}}{(a-18)^{2}} & 0 & \frac{16 a^{2}(a-6) h^{2}}{(a-18)^{2}} \\
\frac{a+6}{8} & 0 & \frac{6-a}{4} & 0
\end{array}\right) \\
& \mathcal{M}_{\gamma_{\varepsilon}^{I V}(l)}=\left(\begin{array}{cccc}
0 & \frac{16 a h^{2}}{9} & 0 & -\frac{8 a h^{2}}{9} \\
-\frac{3(a-2)}{4} & 0 & -\frac{3(a-2)}{8} & 0 \\
0 & -\frac{8 a h^{2}}{9} & 0 & \frac{16 a h^{2}}{9} \\
-\frac{3(a-2)}{8} & 0 & -\frac{3(a-2)}{4} & 0
\end{array}\right) \text {, } \\
& \mathcal{M}_{\gamma_{\varepsilon}^{V}(l)}=\left(\begin{array}{cccc}
\frac{a h}{2 \sqrt{3}} & -\frac{8 a h^{2}}{9} & \frac{a h}{\sqrt{3}} & \frac{4 a h^{2}}{9} \\
-\frac{3(a-4)}{8} & -\frac{a h}{2 \sqrt{3}} & -\frac{3(a-4)}{16} & \frac{a h}{\sqrt{3}} \\
-\frac{a h}{\sqrt{3}} & \frac{4 a h^{2}}{9} & -\frac{a h}{2 \sqrt{3}} & -\frac{8 a h^{2}}{9} \\
-\frac{3(a-4)}{16} & -\frac{a h}{\sqrt{3}} & -\frac{3(a-4)}{8} & \frac{a h}{2 \sqrt{3}}
\end{array}\right) \\
& \mathcal{M}_{\gamma_{\varepsilon}^{V I}(l)}=\left(\begin{array}{cccc}
-\frac{a h}{2 \sqrt{3}} & -\frac{8 a h^{2}}{9} & -\frac{a h}{\sqrt{3}} & \frac{4 a h^{2}}{9} \\
-\frac{3(a-4)}{8} & \frac{a h}{2 \sqrt{3}} & -\frac{3(a-4)}{16} & -\frac{a h}{\sqrt{3}} \\
\frac{a h}{\sqrt{3}} & \frac{4 a h^{2}}{9} & \frac{a h}{2 \sqrt{3}} & -\frac{8 a h^{2}}{9} \\
-\frac{3(a-4)}{16} & \frac{a h}{\sqrt{3}} & -\frac{3(a-4)}{8} & -\frac{a h}{2 \sqrt{3}}
\end{array}\right) \\
& \mathcal{M}_{\gamma_{\varepsilon}^{V I I}(l)}=\left(\begin{array}{cccc}
0 & 2 a h^{2} & -\frac{\sqrt{3(a-2)(a+6)} L}{4} & 0 \\
-\frac{3(a-2)}{4} & 0 & -\frac{3(a-2)}{8} & 0 \\
0 & 0 & \frac{\sqrt{3(a-2)(a+6)} h}{2} & 0 \\
-\frac{3(a-2)}{8} & \frac{\sqrt{3(a-2)(a+6)} h}{4} & -\frac{3(a-2)}{8} & -\frac{\sqrt{3(a-2)(a+6) h}}{2}
\end{array}\right) .
\end{aligned}
$$

The characteristic polynomial $\mathcal{P}_{\gamma_{\varepsilon}^{\mathcal{I}}(l)}(X)$ of the matrix $\mathcal{M}_{\gamma_{\varepsilon}^{\mathcal{I}}(l)}$ is a biquadratic polynomial of the form

$$
\mathcal{P}_{\gamma_{\varepsilon}^{\mathcal{I}}(l)}(X)=X^{4}+C_{\mathcal{I}}(a) X^{2}+D_{\mathcal{I}}(a) .
$$

If the polynomial (8) has purely imaginary roots the equilibrium point $\mathbf{p}_{0}$ is linearly stable. A necessary and sufficient condition for the existence of roots $i \omega_{1},-i \omega_{1}, i \omega_{2}$, $-i \omega_{2}$ with $\omega_{1}, \omega_{2}>0$ and $\omega_{1} \neq \omega_{2}$, is that the following inequalities are verified

$$
C_{\mathcal{I}}(a)>0, D_{\mathcal{I}}(a)>0, C_{\mathcal{I}}(a)^{2}-4 D_{\mathcal{I}}(a)>0 .
$$

If $\omega_{1}=\omega_{2}$ (i.e. when $C_{\mathcal{I}}(a)^{2}-4 D_{\mathcal{I}}(a)=0$ ) then $\mathbf{p}_{0}$ is linearly stable if the matrix $\mathcal{M}_{\gamma_{\varepsilon}^{\mathcal{I}}(l)}$ is diagonalizable. In the table below, we present the expressions involved

\begin{tabular}{|c|c|c|c|}
\hline Family $\mathcal{I}$ & $C_{\mathcal{I}}(a)$ & $D_{\mathcal{I}}(a)$ & $C_{\mathcal{I}}(a)^{2}-4 D_{\mathcal{I}}(a)$ \\
\hline$I, I I, I I I$ & $\frac{2 h^{2} a(5 a+6)(a-6)^{2}}{(a-18)^{2}}$ & $\frac{9 h^{4}(a-6) a^{2}(a-2)^{2}\left(a^{2}-36\right)}{(a-18)^{3}}$ & $\frac{64 h^{4} a^{2}(a-6)^{2}\left(\left(a^{2}-3 a+18\right)^{2} L^{4}\right.}{(a-18)^{4}}$ \\
\hline$I V$ & $2 h^{2} a(a-2)$ & $\frac{h^{4} a^{2}(a-2)^{2}}{4}$ & 0 \\
\hline$V, V I$ & $2 h^{2} a$ & $\frac{h^{4} a^{2}(a-2)^{2}}{8}$ & $h^{4} a^{3}(4-a)$ \\
\hline$V I I$ & $\frac{3 h^{2}(a-6)(a-2)}{4}$ & $-\frac{9 h^{4} a(a+6)(a-2)^{2}}{8}$ & $\frac{8 h^{4}\left(a^{2}-4\right)^{2}}{16}$ \\
\hline
\end{tabular}

For $\gamma_{\varepsilon}^{I V}(l)$ the roots of $\mathcal{P}_{\gamma_{\varepsilon}^{I}(l)}(X)$ are $\pm i \omega= \pm i \sqrt{a(a-2)} h$ with multiplicity two, and the matrix $\mathcal{M}_{\gamma_{\varepsilon}^{I V}(l)}$ is diagonalizable. 
The remaining statements of Theorem 3 are obtained by studying the inequalities (9). This completes the proof of Theorem 3.

\section{ACKNowledgements}

The first, second and fourth author of this work was partially supported by MICINN/ FEDER grant number MTM2011-22587, MINECO grant number MTM2014-51891-P and Fundación Séneca de la Región de Murcia grant number 19219/PI/14. The third author is partially supported by a MINECO grant MTM2013-40998-P, an AGAUR grant number 2014SGR-568, and the grants FP7-PEOPLE-2012-IRSES 318999 and 316338 .

\section{References}

[1] R. Abraham, J.E. Marsden And T. Ratiu, Manifolds, tensor analysis and applications, Second edition. Applied Mathematical Sciences 75, Springer-IIerlag, New York, 1988.

[2] M. Arribas, A. Elipe, L. Floria and A. Riaguas, Oscillators in resonance p:q:r, Chaos, Solitons \& Fractals, 27 (2006), 1220-1228.

[3] N.D. CARAnicolas, 1:1:1 resonant periodic orbits in $3+$ dimensional galactic-type potentials, Astronomy \& Astrophysics 282 (1994), 34-36.

[4] A. Deprit And A. Elipe, The Lissajous transformations II - Normalization, Celestial Mechanics and Dynamical Astronomy 51 (1991), 227-250.

[5] A. Deprit And A. Elipe, Oscillators in resonance, Mechanics Research Communications 26 (1999), 635-640.

[6] A. Elipe, Complete reduction of oscillators in resonance p:q, Physical Review E 61 (2000), 6477-6484.

[7] J.L.G. Guirao, J. Llibre and J.A. Vera, Periodic orbits of Hamiltonian systems: Applications to perturbed Kepler problems, Chaos, Solitons and Fractals 57 (2013), 105-111.

[8] J. Llibre, R. Moeckel and C. Simó, Central Configurations, Periodic Orbits and Hamiltonian Systems, to appear in the Advanced Courses Series of the CRM Barcelona, Birkhäuser, 2015.

[9] J. Llibre, D. PaşCa, C. IIAlls, Periodic solutions of a galactic potential, Chaos, Solitons and Fractals 61 (2014), 38-43.

[10] E.E. Zotos, A new dynamical model for the study of galactic structure, New Astronomy 16 (2011), 391-401.

[11] E.E. Zотоs, Disks controlling chaos in a 3D dynamical model for elliptical galaxies, Baltic Astronomy 20 (2011), 77-90.

[12] E.E. Zотоs, A new dynamical indicator for chaos detection in galactic Hamiltonian systems, Mathematics in Engineering, Science and Aerospace Mesa 3 (2012), 163-178.

[13] E.E. Zотоs, Revealing the evolution, the stability, and the escapes of families of resonant periodic orbits in Hamiltonian systems, Nonlinear Dynam. 73 (2013), 931-962

[14] E.E. Zotos And N.D. Caranicolas, Are semi-numerical methods an effective tool for locating periodic orbits in 3D potentials?, Nonlinear Dyn. 70 (2012), 279-287.

[15] E.E. Zotos And D.D. Carpintero, Orbit classification in the meridional plane of a disk galaxy model with a spherical nucleus, Celest. Mech. Dyn. Astr. 116 (2013), 417-438.

1 Departamento de Matemática Aplicada, Universidad de Salamanca, C/del Parque, 2, 37008 Salamanca (Castilla y León) Spain.

E-mail address: tbustos@usal.es

2 Departamento de Matemática Aplicada y Estadística. Universidad Politécnica de Cartagena, Hospital de Marina, 30203-Cartagena, Región de Murcia, Spain.-Corresponding AUTHOR-

E-mail address: juan.garcia@upct.es

3 Departament de Matemàtiques. Universitat Autònoma de Barcelona, Bellaterra, 08193-Barcelona, Catalonia, Spain

E-mail address: jllibre@mat.uab.cat 
4 Centro Universitario de la Defensa. Academia General del Aire. Universidad Politécnica de Cartagena, 30720-Santiago de la Ribera, Región de Murcia, Spain E-mail address: juanantonio.vera@cud.upct.es 